\title{
EFFECT OF PRESSURE ON THE SOLITARY WAVE PROPAGATION IN ONE-DIMENSIONAL LATTICE
}

\author{
R.KH. SABIROV \\ Faculty of Physics, V.I. Lenin State Pedagogical University, 119435 Moscow, Russia \\ (Received May 20, 1991; in final form February 27, 1992) \\ The solitary wave propagation in an anharmonic monoatomic linear \\ chain for the case of an external pressure is investigated. The potential en- \\ ergy of the crystal with anharmonicities of the third and fourth orders is \\ considered. Two kinds of solitary waves are obtained. The first kind corre- \\ sponds to the compressive soliton and the second one - to the rarefactive \\ soliton. In the case of the lattice with the cubic anharmonicity the exact \\ theoretical study was done. Numerical calculations were performed for the \\ general case. It is found that when pressure rises, amplitudes of solitons also \\ increase.
}

PACS numbers: $63.20 . R y$

\section{Introduction}

Wadati [1] has investigated the solitary and periodic waves propagation in the nonlinear lattice. As a model, Wadati has adopted the one-dimensional lattice, which was constituted by $N$ particles of mass $m$ connected by nonlinear springs with cubic and quartic nonlinearities. In the previous papers $[2,3]$ we have studied the solitary waves or solitons propagation in a one-dimensional chain under constant external forces deforming the lattice. In the case of external tensions the properties of the soliton solutions are significantly modified. In particular, as shown in [3], for the discussed there relations between the interatomic-potential constants and for prescribed external tensions, the soliton solution does not exist. In other words, the soliton amplitude is equal to zero in this case.

In the present paper we consider the solitary wave propagation in the anharmonic monoatomic linear chain with the nearest-neighbour interaction for the case of an arbitrary external pressure. As we shall see later, the properties of solitons existing in a one-dimensional lattice significantly depend on whether the lattice is under the pressure or under the tension. The problems will be examined below in more detail. 


\section{Potential energy of the system}

The potential energy of this system generaly can be written in the form

$$
V_{\mathrm{pot}}=\sum_{n=1}^{N} \Phi\left(R_{n, n-1}\right)+V_{1}, \quad R_{n, n-1}=R_{n}-R_{n-1},
$$

where $\Phi\left(R_{n, n-1}\right)$ is the interaction potential between neighbouring atoms, $R_{n}$ is the position vector for the atom in the lattice site $n$. Let us consider the case when the external pressure is fixed. Then, for the one-dimensional chain the effect of the external forces can be described by the external pressure $P$ which acts on the ends of the chain [4]

$$
V_{1}=P\left(R_{N}-R_{0}\right)=P \sum_{n=1}^{N} R_{n, n-1} .
$$

We consider the potential energy of the crystal with anharmonicities of the third and fourth orders in the series expansion of the potential energy. Thus the expression $\Phi\left(R_{n, n-1}\right)$ is

$$
\begin{aligned}
& \Phi\left(R_{n, n-1}\right)=\Phi(a)+\alpha\left(R_{n, n-1}-\alpha\right)^{2}-\beta\left(R_{n, n-1}-\alpha\right)^{3} \\
& +\gamma\left(R_{n, n-1}-\alpha\right)^{4}
\end{aligned}
$$

where $\Phi(a)$ is the depth of the potential, $a$ is the average distance between neighbouring atoms in the harmonic approximation at $P=0$ and $\alpha, \beta, \gamma$ are the coefficients defining the interaction of atoms. Throughout the paper, we assume $\alpha, \beta, \gamma \geq 0$. The second term on the right hand side of (2.3) is the harmonic approximation; the third and fourth terms are corrections due to anharmonicity to the second term. In the harmonic approximation $\beta, \gamma=0$.

Following $[2,5]$, we can obtain

$$
\begin{aligned}
& \Phi\left(R_{n, n-1}\right)+P R_{n, n-1}=-\Phi_{0}\left(a_{0}\right)+k_{2}\left(R_{n, n-1}-a-a_{0}\right)^{2} \\
& +k_{3}\left(R_{n, n-1}-a-a_{0}\right)^{3}+\gamma\left(R_{n, n-1}-a-a_{0}\right)^{4},
\end{aligned}
$$

where $\Phi_{0}\left(a_{0}\right)$ is the constant and it describes the depth of the interatomic potential at $P \neq 0$. The parameters $k_{2}, k_{3}$ and $a_{0}$ can be written as

$$
k_{2}=1-3 y+6 b y^{2}, k_{3}=-1+4 b y, a_{0}=\frac{\alpha}{\beta} y,
$$

where $b=\alpha \gamma / \beta^{2}$. The value $y$ can be determined from the equation

$$
12 b y^{3}-9 y^{2}+6 y+\frac{P}{P_{0}}=0, P_{0}=\frac{\alpha^{2}}{3 \beta} \text {. }
$$

Equation (2.6) has three solutions for $y(P)$ with $P$ being an argument. However, there is only one real solution which from "physical" prerequisites can be accepted, i.e. such that $y(P) \rightarrow 0$ as $P \rightarrow 0$.

Now introducing the dynamical displacements of the atoms $u_{n}$, according to

$$
R_{n, n-1}=a+a_{0}+u_{n}-u_{n-1} \text {, }
$$


the potential energy of the lattice can be written as

$$
V_{\text {pot }}=\sum_{n=1}^{N}\left[k_{2}\left(u_{n}-u_{n-1}\right)^{2}+k_{3}\left(u_{n}-u_{n-1}\right)^{3}+\gamma\left(u_{n}-u_{n-1}\right)^{4}\right] \text {. }
$$

Formally the functional form of the potential energy of the lattice (2.8) coincides with that obtained for the free lattice, but in our case the parameters $k_{2}$ and $k_{3}$ are pressure dependent.

\section{Solitary wave solution}

In this section we get the soliton solutions for the lattice model with the potential energy in the form considered in the previous Section. The equation of motion of the $n$-th atom can be written as

$$
m \ddot{u}_{n}=2 k_{2}\left(\Delta_{n+1}-\Delta_{n}\right)+3 k_{3}\left(\Delta_{n+1}^{2}-\Delta_{n}^{2}\right)+4 \gamma\left(\Delta_{n+1}^{3}-\Delta_{n}^{3}\right),
$$

where $\Delta_{n}=u_{n}-u_{n-1}$ and the dot stands for differentiation with respect to time $t$. Being interested in solutions either of the smooth waves-type, or of the waves with the long wave length compared with the spacing of atoms in the lattice, one can adopt the limit of a continuum model. Expressing the displacements $u_{n \pm 1}$ in the terms of the displacement $u_{n}$ and its spatial derivatives by means of the Taylor series, we obtain a new form of the equation of motion

$$
\ddot{u}=v^{2}\left[u^{\prime \prime}+\frac{1}{12} l^{2} u^{\prime \prime \prime \prime}+3 l \frac{k_{3}}{k_{2}} u^{\prime} u^{\prime \prime}+6 l^{2} \frac{\gamma}{k_{2}} u^{\prime \prime}\left(u^{\prime}\right)^{2}\right],
$$

where $l=a+a_{0}, v^{2}=2 k_{2} l^{2} \alpha / m$ and the primes denote the partial differentiations with respect to space coordinate. It is noteworthy that expression (3.2) was gained in the approximation when only the derivatives with respect to space coordinate up to the fourth order have been taken into account. Moreover, we assume an infinite number of atoms in the chain.

The travelling wave solution (solitary wave) corresponding to Eq. (3.2) can be obtained putting $u(x, t)=u(x-V t)$ with $\ddot{u}=V^{2} u^{\prime \prime}$. Here $V$ is the velocity of the travelling wave in the lattice. Then, integrating Eq. (3.2) twice with respect to $x$, we obtain

$$
\frac{\mathrm{d} Z}{\mathrm{~d} x}= \pm\left(-A Z^{4}-B Z^{3}+C Z^{2}+c_{1} Z+c_{2}\right)^{1 / 2}, \quad Z=u^{\prime},
$$

where $c_{1}$ and $c_{2}$ are arbitrary constants and

$$
A=12 \frac{\gamma}{\alpha k_{2}}, \quad B=12 \frac{\beta k_{3}}{\alpha \ell k_{2}}, \quad C=\frac{12}{\ell^{2}}\left(\frac{V^{2}}{v^{2}}-1\right) .
$$

The soliton solution is obtained under the conditions: $Z \rightarrow 0$ and $Z_{x}^{\prime} \rightarrow 0$ as $|x| \rightarrow \infty$. This limiting condition holds if in Eq. (3.3) $c_{1}=0, c_{2}=0$ and $C>0$. Following the treatment of Wadati [1] and making use of the work [2], we can obtain two kinds of solitary waves. The first one corresponds to the compressive soliton and the second one to the rarefactive soliton. These soliton solutions are given by

$$
Z=-\frac{C}{A}\left\{-Z_{2} \operatorname{sh}^{2}\left[\frac{1}{2} \sqrt{C}\left(x+x_{0}\right)\right]+Z_{1} \operatorname{ch}^{2}\left[\frac{1}{2} \sqrt{C}\left(x+x_{0}\right)\right]\right\}^{-1}
$$


and

$$
Z=\frac{C}{A}\left\{Z_{1} \operatorname{sh}^{2}\left[\frac{1}{2} \sqrt{C}\left(x+x_{0}\right)\right]-Z_{2} \operatorname{ch}^{2}\left[\frac{1}{2} \sqrt{C}\left(x+x_{0}\right)\right]\right\}^{-1},
$$

respectively. $x_{0}$ is the integration constant. The constants $Z_{1}$ and $Z_{2}$ can be rewritten as

$$
Z_{1}=\frac{1}{2 A}\left(|B|+\sqrt{B^{2}+4 A C}\right), \quad Z_{2}=\frac{1}{2 A}\left(|B|-\sqrt{B^{2}+4 A C}\right) .
$$

The expressions, similar to (3.5) and (3.6), were obtained previously in [1], but in our case parameters $A, B$ and $C$ depend on the pressure $P$. The exact solitary wave solutions (3.5) and (3.6) are equivalent to the results in [1] for $P=0$. It should be noted that Eqs. (3.5) and (3.6) can also be obtained formally from [2,3] if substituting $P=-F$, where $F$ is the external tension. In general, $x$ in Eqs. (3.5) and (3.6) linearly depends on the space coordinate $x^{\prime}$ and time $t$ by $x=x^{\prime}-V t$.

It should be emphasized that the soliton solutions (3.5) and (3.6) were derived under the condition that the parameter $B \leq 0$. If we change the sign in Eq. (3.5) before the curly bracket to the opposite one, we obtain the solitary solution (rarefactive soliton) for the case $B \geq 0$. Doing similar manipulation Eq. (3.6) can be written in the form of the solitary solution for the compressive soliton.

The expression $Z(x)$ characterizes the local deformation of the atomic linear chain, $Z=\mathrm{d} u / \mathrm{d} x$, where $u$ is the displacement from the equilibrium positions of atoms. According to [1] the function $u$ can be written in the form $(B \leq 0)$

$$
u=\varepsilon \frac{2}{\sqrt{A}} \operatorname{arctg}\left\{\left(\frac{\sqrt{B^{2}+4 A C}+\varepsilon|B|}{\sqrt{B^{2}+4 A C}-\varepsilon|B|}\right)^{1 / 2} \operatorname{th}\left[\frac{1}{2} \sqrt{C}\left(x+x_{0}\right)\right]\right\}+u_{1},
$$

where $u_{1}$ is the integration constant, $\varepsilon= \pm 1$ for the compressive and rarefactive solitons, respectively.

\section{The case of the cubic anharmonicity}

As the first step we consider the case of $\gamma=0$ in (2.3) and (2.8), since this case is the simplest one. Determining $k_{2}$ and $k_{3}$ by (2.5) and (2.6) we obtain

$$
Z=\frac{C}{B} \operatorname{ch}^{-2}\left[\frac{1}{2} \sqrt{C}\left(x+x_{0}\right)\right], u=-\frac{2 \sqrt{C}}{B}\left[1-\operatorname{th}\left(\frac{1}{2} \sqrt{C}\left(x+x_{0}\right)\right)\right],
$$

where

$$
B=-12 \frac{\beta}{\alpha \ell}\left(1+3 \frac{\beta}{\alpha^{2}} P\right)^{-1 / 2} .
$$

It is seen from (4.2) that $B<0$. The value $u$ given by (4.1) describes the step-like transitions from the value $u_{0}=-4 \sqrt{C} / B$ at $x=-\infty$ to zero at $x=\infty$. Such step-like transition is usually denoted as a kink. This kink moves with velocity $V$ in the $x$-direction. In (4.1) we take into account the boundary condition $u(\infty)=0$.

As seen from Eq. (4.1) for the considered case, we have

$$
\Delta x=\frac{4 \pi}{\sqrt{C}}=\frac{2 \pi}{\sqrt{3}} \ell\left(\frac{V^{2}}{v^{2}}-1\right)^{-1 / 2}
$$




$$
Z_{0}=\frac{C}{B}=\frac{\alpha}{\beta \ell}\left(\frac{V^{2}}{v^{2}}-1\right)\left(1+3 \frac{\beta}{\alpha^{2}} P\right)^{1 / 2},
$$

where $\Delta x$ and $Z_{0}$ are the width and amplitude of the compressive soliton, respectively. We want to point out that in this case the solitary wave solution contains only the compressive soliton. Explicitly, we have that

$$
v^{2}=2 \frac{\alpha \ell^{2}}{m}\left(1+3 \frac{\beta}{\alpha^{2}} P\right)^{1 / 2}, \quad \ell=a+\frac{\alpha}{3 \beta}\left(1-\sqrt{1+3 \frac{\beta}{\alpha^{2}} P}\right) .
$$

It is convenient to write the equality $u_{0}=-4 \sqrt{C} / B$ in the form

$$
\left(\frac{V^{2}}{v^{2}}-1\right)^{1 / 2}=\frac{\sqrt{3} \beta}{2 \alpha} u_{0}\left(1+3 \frac{\beta}{\alpha^{2}} P\right)^{-1 / 2} \text {. }
$$

This equation defines the dependence of the soliton velocity $V$ on the pressure $P$ and the amplitude of the kink $u_{0}$. Substituting (4.6) into (4.3) and (4.4) we find

$$
\begin{aligned}
& \Delta x=\frac{4 \pi \alpha \ell}{3 \beta u_{0}}\left(1+3 \frac{\beta}{\alpha^{2}} P\right)^{1 / 2}, \\
& Z_{0}=\frac{3 \beta u_{0}^{2}}{4 \alpha \ell}\left(1+3 \frac{\beta}{\alpha^{2}} P\right)^{-1 / 2} .
\end{aligned}
$$

It is easy to verify that $Z_{0} \Delta x=\pi u_{0}$.

Let us consider the solitons with $u_{0}=$ const. The velocity of the soliton $V$ according to (4.6) decreases as the pressure $P$ increases. The same conclusion can be drawn with respect to the soliton amplitude $Z_{0}$. However, the width of the compressive soliton increases with the pressure $P$. Roughly speaking, pressure induces the destruction of the soliton with $u_{0}=$ const. It should be noted that in the case of the external tension action an opposite tendency takes place, the tension causes the compressive soliton to be more stable.

All the above formulae have been derived in the case of the continuous approximation. This approximation is valid if $\Delta x \gg \ell$ and

$$
\left(1+3 \frac{\beta}{\alpha^{2}} P\right)^{1 / 2} \gg \frac{3 \beta u_{0}}{4 \pi \alpha}
$$

For a given pressure $P$ this inequality imposes a restriction on the possible values $u_{0}$ and then according to (4.6) also on the velocity of a soliton $V$.

\section{General treatment}

Let us now consider the general case when $\gamma \neq 0$. In this case the compressive . and rarefactive solitons can propagate in the lattice at the same time. The sign of $B$ is essential since it determines the form of solitary solutions for the compressive and rarefactive solitons. This sign coincides with the sign of $k_{3} / k_{2}$. The ratio $k_{3} / k_{2}$ is determined by Eqs. (2.5) and (2.6). Because of physical reasons we shall confine ourselves to the solution $y(P)$ of Eq. (2.6) which tends to zero if $P \rightarrow 0$. Then it is not difficult to show that $y(P) \leq 0$ and according to (2.5) $k_{2}>0$ and $k_{3}<0$. 


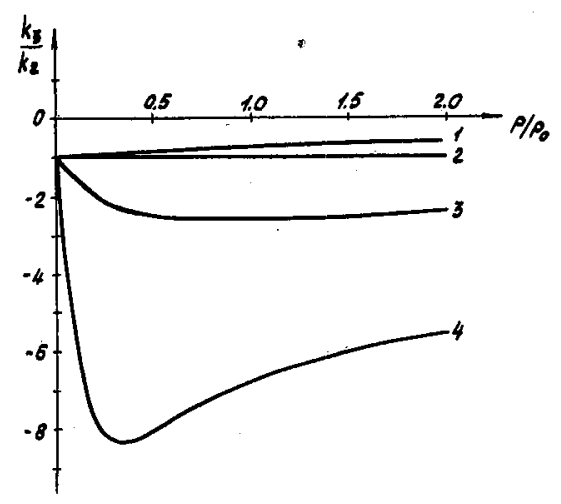

Fig. 1. The dependence of the ratio $k_{3} / k_{2}$ on the reduced pressure $P / P_{0}:(1) b=0.01$, 0.1 ; (2) $b=1$; (3) $b=10$; (4) $b=100$.

Therefore, we can write $B<0$ regardless of $P$. The results of the calculation $k_{3} / k_{2}$ are shown in Fig. 1. It should be noted that in the case of the tension force acting on the lattice, the parameter $B$ may change its sign to the opposite one if $b>3 / 8$. Here for $b<3 / 8$, the parameter $B<0$.

For the case of $B \leq 0$ the solitary solutions are given by Eqs. (3.5) and (3.6) for the compressive and rarefactive solitons, respectively. Let us consider the dependence of the amplitude of the soliton $Z^{*}=(\beta l / \alpha)|Z(0)|$ on the pressure $P$. According to (3.5) and (3.6) the function $Z^{*}$ is

$$
Z^{*}=\frac{2\left|k_{2} / k_{3}\right|\left(V^{2} / v^{2}-1\right)}{ \pm 1+\sqrt{1+4 b\left(k_{2} / k_{3}^{2}\right)\left(V^{2} / v^{2}-1\right)}}
$$

where the upper sign refers to the compressive and the lower sign to the rarefactive solitons.

If $4 b k_{2}\left(V^{2} / v^{2}-1\right) \gg k_{3}^{2} \mathrm{Eq}$. (5.1) has the form

$$
Z^{*}=\left[\frac{k_{2}}{b}\left(\frac{V^{2}}{v^{2}}-1\right)\right]^{1 / 2} \text {. }
$$

Thus in this case the amplitudes of the compressive and rarefactive solitons are equal.

For pressures where $4 b k_{2}\left(V^{2} / v^{2}-1\right) \ll k_{3}^{2}$ and for the compressive soliton, $Z^{*}$ has the following form:

$$
Z^{*}=\left|\frac{k_{2}}{k_{3}}\right|\left(\frac{V^{2}}{v^{2}}-1\right) \text {. }
$$

At the same time the quantity $Z^{*}$ for the rarefactive soliton can be written as follows:

$$
Z^{*}=\left|k_{3}\right| / b \text {. }
$$

From Eqs. (5.3) and (5.4) we conclude that the amplitudes of the compressive and rarefactive solitons are not equal. 
As one should expect from (2.5) and (2.6) $\left|k_{2}\right|$ and $\left|k_{3}\right|$ increase with the pressure $P$. The dependence of ratio $k_{3} / k_{2}$ on the pressure $P$ is shown in Fig. 1 . The ratio $k_{2} / k_{3}$ appearing in (5.3) tends to infinity as $P \rightarrow \infty$. However, the condition $4 b k_{2} / k_{3}^{2}=1.5$ reaches for $P=\infty$. It means that in high pressure regime and as long as the velocity of soliton significantly differs from zero, its amplitude is given by Eq. (5.2).

Now we discuss the numerical results for the pressure dependence of the value $Z^{*}$ for the compressive and rarefactive solitons. The graphs of the function $Z^{*}(P)$ for different parameters $b$ and $V$ in the region where $2 P_{0} \geq P \geq 0$ are given in Figs. 2 and 3. It can be seen that the pressure dependence of $Z^{*}$ is approximately
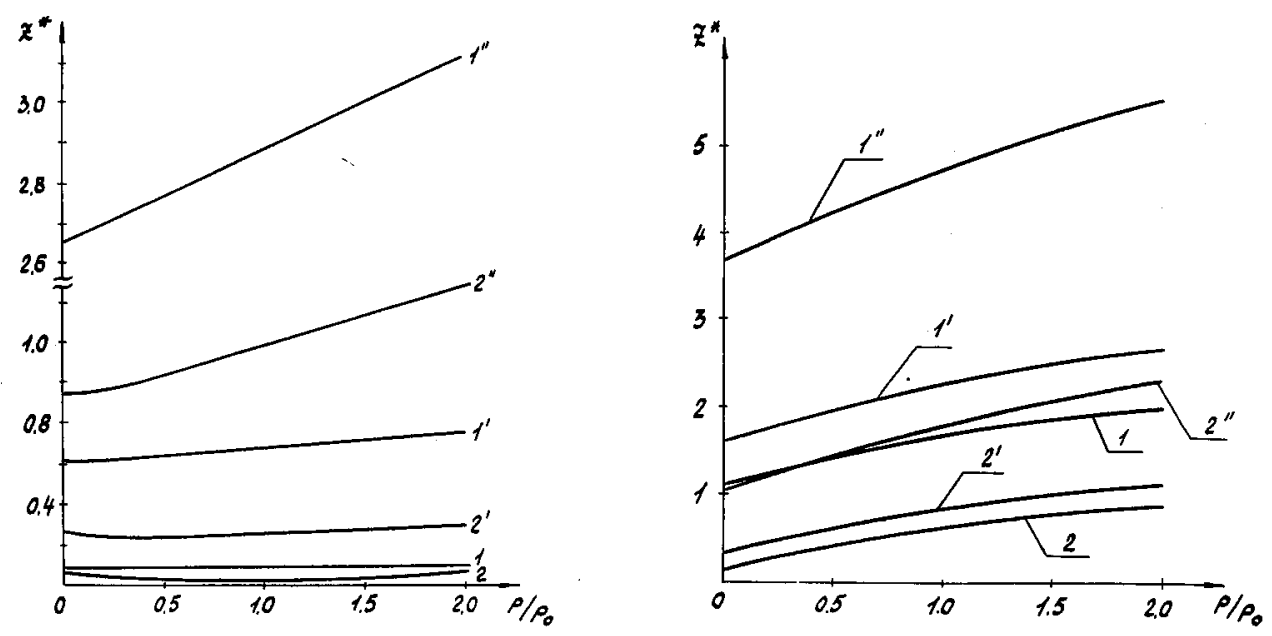

Fig. 2. The dependence of the value $Z^{*}$ on the reduced pressure $P / P_{0}$ for the compressive soliton: (1) $b=1, V^{2} / v^{2}=1.1,\left(1^{\prime}\right) b=1, V^{2} / v^{2}=2,\left(1^{\prime \prime}\right) b=1, V^{2} / v^{2}=11$, (2) $b=10, V^{2} / v^{2}=1.1,\left(2^{\prime}\right) b=10, V^{2} / v^{2}=2,\left(2^{\prime \prime}\right) b=10, V^{2} / v^{2}=11$.

Fig. 3. The dependence of the value $Z^{*}$ on the reduced pressure $P / P_{0}$ for the rarefactive soliton: (1) $b=1, V^{2} / v^{2}=1.1,\left(1^{\prime}\right) b=1, V^{2} / v^{2}=2,\left(1^{\prime \prime}\right) b=1, V^{2} / v^{2}=11$, (2) $b=10, V^{2} / v^{2}=1.1,\left(2^{\prime}\right) b=10, V^{2} / v^{2}=2,\left(2^{\prime \prime}\right) b=10, V^{2} / v^{2}=11$.

proportional to $P$, except the region of small $P$ for the compressive soliton. In this region, the amplitude $Z^{*}$ at the beginning decreases from its value at $P=0$ down to some minimum and then it rises. This tendency is well-pronounced for larger $b$ and smaller $V$.

From Figs. 2 and 3 it is seen that amplitudes of the compressive and rarefactive solitons increase with $P$. For some values of $b$ and $V$, the amplitude of the rarefactive soliton is larger than the amplitude of the compressive soliton. This fact is more noticeable in the case of small $b$ and $V$. Therefore we expect a small compressive soliton and a large rarefactive soliton. As our calculations show, the action of pressure on a lattice makes the solitons more stable, leading also to the increase of the soliton amplitudes. 


\section{Conclusion}

Having analysed the results of this paper and results obtained in papers [2,3] we can conclude the following: the properties of solitons in the lattice depend significantly on the kind of force deforming the lattice. In connection with this conclusion we can notice the basic results concerning the properties of the solitons in the lattice under the tension.

In this case the properties of the solitons depend significantly on the value of the characteristic parameter $b(2.5)$. If $b<3 / 8$, then the amplitudes of the compressive and rarefactive solitons decrease with the rise of the external tension force $F$. For some certain value of $F$, the solitons in the form of the compressive solitary wave vanish. On the other hand, if $b>3 / 8$, then the amplitudes of the compressive and rarefactive solitons are rising with the rising value of $F$. In this case, when the certain value of $F=F^{*}$ is chosen, the amplitudes of these solitons are equal. The amplitude of the compressive soliton becomes larger than the amplitude of rarefactive soliton when $F>F^{*}$. It should be noted, however, that the amplitude of the rarefactive soliton is larger than the amplitude of the compressive soliton in the limit $F=0$. At $b=3 / 8$ one can find the certain value of $F$ when any kinds of the solitary waves are absent in the lattice.

\section{References}

[1] M. Wadati, J. Phys. Soc. Jpn. 38, 673 (1975).

[2] R.Kh. Sabirov, Fiz. Tverd. Tela 31, 167 (1989).

[3] R.Kh. Sabirov, Fiz. Tverd. Tela 32, 1992 (1990).

[4] N.M. Plakida, T. Siklos, Phys. Status Solidi 39, 171 (1970).

[5] R.Kh. Sabirov, Fiz. Tverd. Tela 26, 1358 (1984). 